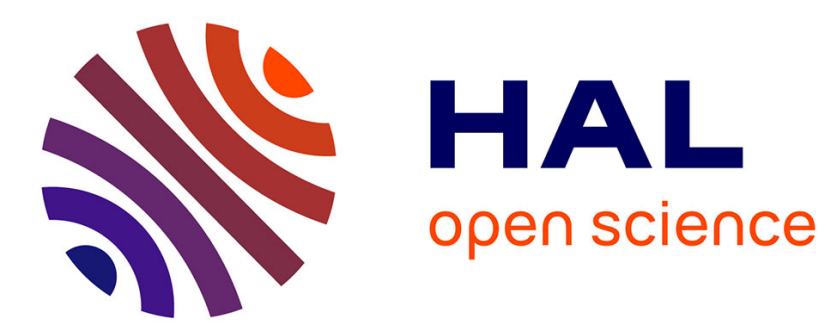

\title{
Weak adhesive junctions
}

P.-G. de Gennes

\section{To cite this version:}

P.-G. de Gennes. Weak adhesive junctions. Journal de Physique, 1989, 50 (18), pp.2551-2562. 10.1051/jphys:0198900500180255100 . jpa-00211081

\section{HAL Id: jpa-00211081 https://hal.science/jpa-00211081}

Submitted on 1 Jan 1989

HAL is a multi-disciplinary open access archive for the deposit and dissemination of scientific research documents, whether they are published or not. The documents may come from teaching and research institutions in France or abroad, or from public or private research centers.
L'archive ouverte pluridisciplinaire HAL, est destinée au dépôt et à la diffusion de documents scientifiques de niveau recherche, publiés ou non, émanant des établissements d'enseignement et de recherche français ou étrangers, des laboratoires publics ou privés. 
Classification

Physics Abstracts

$62.20 \mathrm{P}-68.35 \mathrm{G}$

\title{
Weak adhesive junctions
}

\author{
P. G. de Gennes \\ Collège de France, F-75231 Paris Cedex 05, France
}

(Reçu le 6 mars 1989, accepté le 3 mai 1989)

\begin{abstract}
Résumé. - Parmi les situations de jonction faible, on peut citer : (1) des contacts verre/caoutchouc ; (2) des contacts caoutchouc/caoutchouc, avec faible pontage ; (3) l'interface entre deux polymères $\mathrm{A}$ et $\mathrm{B}$ décoré par un copolymère séquencé $\mathrm{AB} ;(4)$ les situations d'interdiffusion partielle entre deux blocs du même polymère. Quand une fracture se propage le long d'une jonction faible, la dissipation est localisée dans une fine lame près du plan de fracture. Nous analysons le comportement de cette lame avec deux ingrédients principaux : a) la contrainte seuil $\sigma_{\mathrm{c}}$ nécessaire pour démarrer le fluage - lié à des scissions chimiques, dans les cas $(1,2)$ ou à un seuil plastique, pour les polymères vitreux des exemples $(3,4) ; b$ ) un mécanisme de succion, avec une vitesse de succion proportionnelle à la contrainte locale de traction, jusqu'à ce que le volume aspiré $\left(1 \mathrm{~cm}^{2}\right)$ ait atteint une valeur limite $h_{\mathrm{f}}$. En postulant qu'il n'y a pas de cavitation (pas de craquelures), et en effectuant une analyse très rustique du comportement mécanique autour de la jonction, nous sommes conduits à deux régimes mécaniques : $(\alpha)$ quand la vitesse d'avancée de la fracture est en-dessus d'un certain seuil $V^{*}$, on a un comportement quasi statique et l'énergie de fracture est $G_{\mathrm{IC}}=\sigma_{\mathrm{c}} h_{\mathrm{f}} ;(\beta)$ pour $V>V^{*}$, la largeur de la région de succion est très étendue et $G_{\mathrm{IC}}$ croît avec $V$. Mais l'analyse mécanique précise de notre modèle reste à faire.
\end{abstract}

\begin{abstract}
Weak mechanical junctions can be found (1) at glass/rubber interfaces ; (2) between two rubber blocks with a few interblock crosslinks ; (3) between two glassy polymers A/B with a monolayer of $\mathrm{AB}$ copolymer ; (4) between two identical copolymers after partial interdiffusion. When a fracture propagates along such a junction, the dissipation tends to be localized in the junction region. We present a phenomenological description of this process in termes of two ingredients : (a) a threshold stress $\sigma_{\mathrm{c}}$ associated with chemical scission in cases $(1,2)$ and with plastic flow in cases $(3,4) ;(b)$ a « succion " process - with a succion velocity proportional to the local stress $\sigma,-$ ending when the volume transfer (per unit area) has reached a certain limit $h_{\mathrm{f}}$. We restrict (for the moment) our attention to cases without cavitation (no crazes). From a very crude analysis of the mechanical behavior around the weak junction, we are led to expect two fracture regimes: $(\alpha)$ at low fracture velocities $V$, the process is quasi static and the fracture energy $G_{\mathrm{IC}}$ scales like $\sigma_{\mathrm{c}} h_{\mathrm{f}} ; \beta$ ) beyond a velocity $V^{*}$, the length of the succion region is very much spread out, and $G_{\mathrm{IC}}$ increases with $V$. But we have not yet built a precise mechanical analysis of our model.
\end{abstract}

\section{Physical background.}

Cohesive rupture of polymers (rubbers or glassy plastics) often involves a large dissipation around the fracture tip [1]. Sometimes, viscoelastic losses in the bulk of the material may extend rather far out - e.g. with weakly cross linked elastomers [2]. We are concerned here 
with an opposite situation, where the dissipation is restricted to a thin sheet near the fracture plane. Examples are shown in figures 1-4. The first two examples involve rubbers : in these cases, the polymer medium is locally fluid, but cohesion is maintained by cross linking. The last two examples involve glassy polymers, with a high elastic modulus $\mu$ : but, when the local stress exceeds a certain plastic limit $\sigma_{y}$, the material can yield. We restrict our attention to cases where this occurs through chain slippage and not by chain scission.

1.1 RubBERS. - In all cases, a weak junction is characterized by a relatively low number $\nu$ of bridges per unit area. In the glass/rubber case (Fig. 1), $\nu$ is the number of primer molecules (e.g. organosilanes/ which are bound to the glass surface). For the rubber ${ }^{\mathrm{I}} /$ rubber $^{\text {II }}$ contact (Fig. 2), the situation is more subtle : we can have both physical bridges $\left(\nu_{\mathrm{p}}\right)$ (dangling chains from part I penetrating into part II and vice versa) and chemical bridges $\left(\nu_{\mathrm{c}}\right)$ (resulting from a weak curing after contact). For many features of the adhesion process, the sum $\nu=\nu_{\mathrm{p}}+\nu_{\mathrm{c}}$ should be the essential parameter.

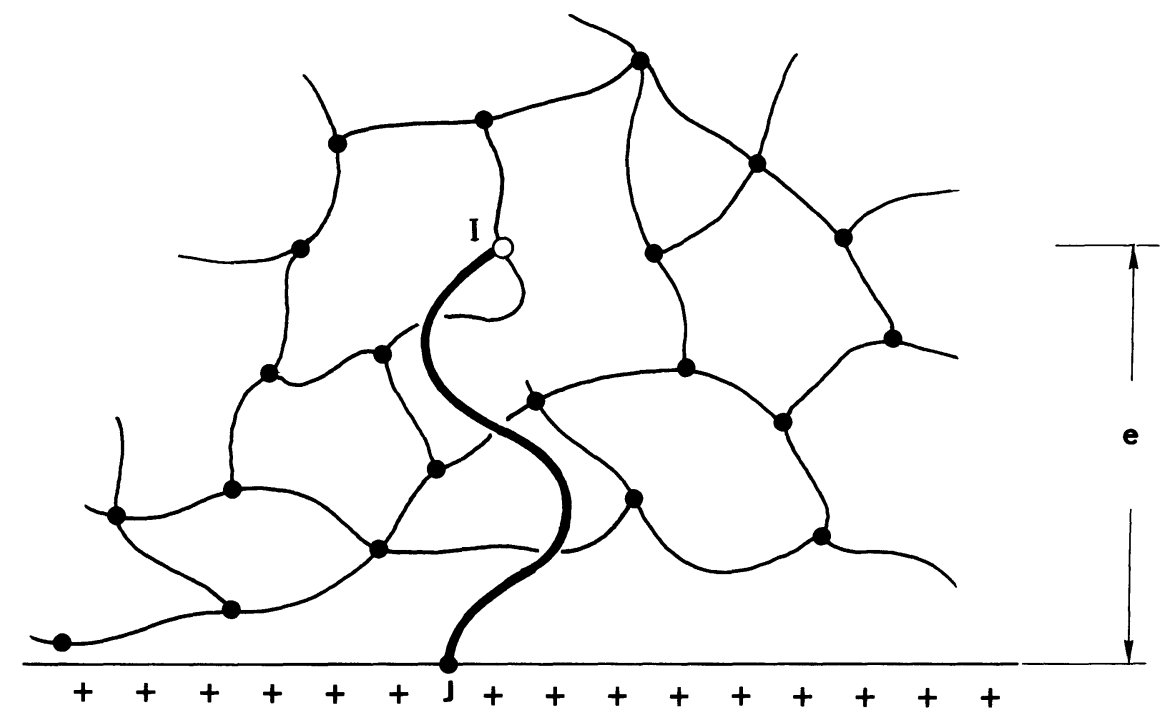

Fig. 1. - A model case for glass/rubber adhesion. The primer molecule (IJ) is assumed to be very long (somewhat longer than practical primers). It is bound chemically at point $\mathrm{J}$ to the glass, and at point I to the rubber network. In the discussion of section 2 , it is assumed that chemical rupture occurs at point $I$. The region (of thickness $e$ ) where the primers are present, is called the "skin " of the junction. The curvilinear length $L_{\mathrm{b}}$ of the bridge is larger than $e$.

Some chemical bonds have to be broken in the fracture process : in the glass/rubber case, we shall assume, for definiteness, that this takes place at the cross link points I between the primer chain and the bulk elastomer. In the rubber/rubber case, this may take place anywhere along the bridge. The chemical reaction leading to rupture will usually be dominated by impurities in the polymer matrix. We expect the reaction rates $1 / \tau_{d}$ to be rapidly increasing with the applied stress $\sigma$. Because the overlap between electron orbitals of adjacent backbone atoms decreases exponentially with distance, we could use a rate law of the form

$$
\frac{1}{\tau_{\mathrm{d}}}=\frac{1}{\tau_{\mathrm{d} 0}} \exp \left(\sigma / \sigma_{\mathrm{d}}\right) \text {. }
$$




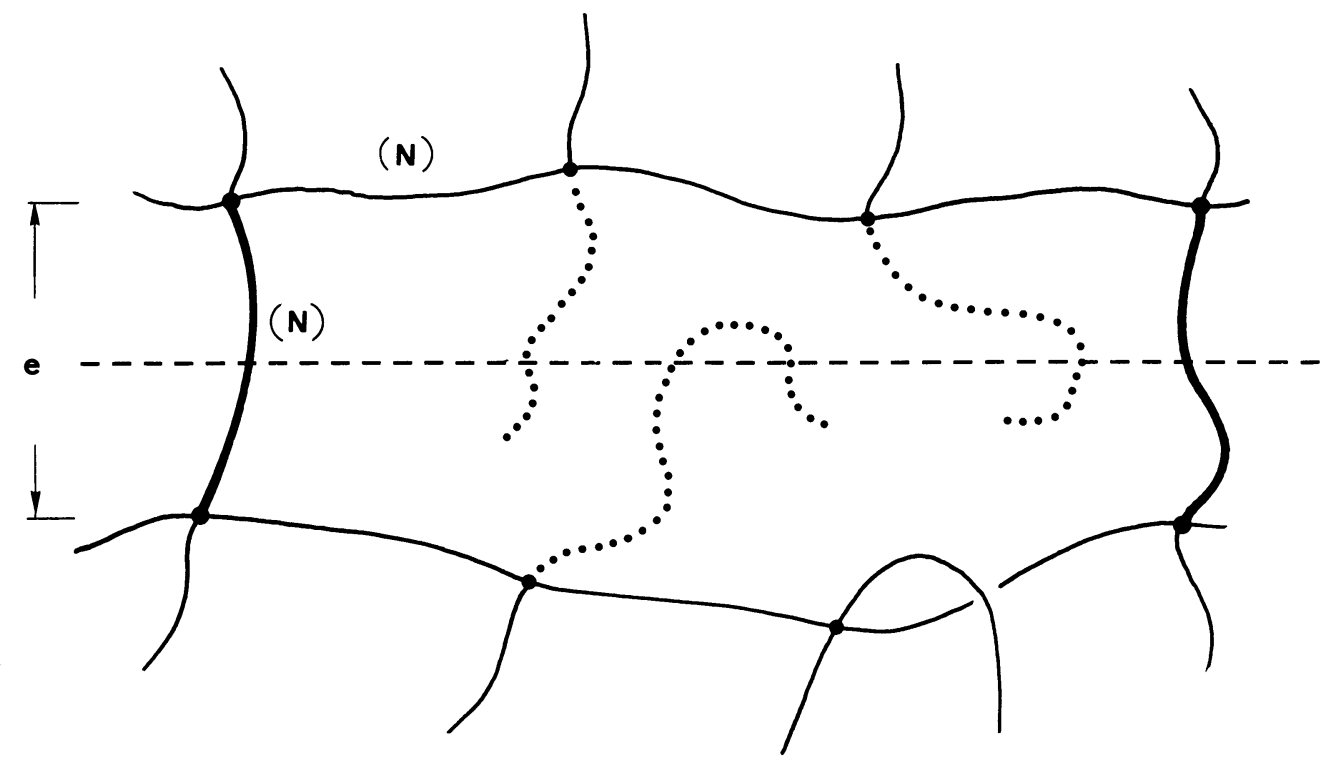

Fig. 2. - A model for a weak rubber/rubber junction. Here the skin thickness $e$ is comparable to the coil size between cross links $\left(N^{1 / 2} a\right)$. The two parts are connected by chemical bridges (thick dark lines) with $\nu_{\mathrm{c}}$ bridges $/ \mathrm{cm}^{2}$, and also by physical bridges (dotted lines) with $\nu_{\mathrm{p}}$ bridges $/ \mathrm{cm}^{2}$. For most of our discussion, the important parameter is the total number $\nu=\nu_{\mathrm{p}}+\nu_{\mathrm{c}}$.

Here, $\tau_{\mathrm{d}}$ is the degradation time for one bond, under a given stress $\sigma . \tau_{\mathrm{d} 0}$ is the corresponding time at rest. $\sigma_{\mathrm{d}}$ is a characteristic stress. Both $\sigma_{\mathrm{d}}$ and $\tau_{\mathrm{d} 0}$ are independent of chain length, but they do depend sensitively on the impurity content. Because of the rapid variation implied by equation (1.1), we may define a chemical yield stress $\sigma_{\mathrm{c}}$ such that $\tau_{\mathrm{d}}$ is comparable to the duration $\tau$ of the stress

$$
\sigma_{\mathrm{c}}=\sigma_{\mathrm{d}} \ln \left(\frac{\tau_{\mathrm{d} 0}}{\tau}\right)
$$

A more precise definition of $\tau$ will be given in section 2. But in practice since it enters (1.2) only through a $\log$, - we may often consider that $\sigma_{\mathrm{c}}$ is a material constant (for a given impurity content).

1.2 Glassy POLYMERS. - For the junctions [polymer A/AB copolymer/polymer B], displayed in figure 3, $\nu$ is the number of copolymer chains per unit area. (This number is not easy to monitor in practice : if we impose a nominal $\nu$ which is small, we may often find that the copolymer aggregates during deposition and achieves $\nu$ values which are high in some regions, and negligible in other parts of the junction.)

Partial interdigitation at $A / A$ contacts (Fig. 4) has been achieved in elegant experiments by Kausch and coworkers [3], where a contact is « healed » (at temperatures slightly above the glass point $T_{\mathrm{g}}$ ) during times $t_{\mathrm{h}}$ smaller than the reptation time $T_{\mathrm{r}}$ [4]. For long chains (number of monomers/chain $N$ larger than the entanglement limit $N_{\mathrm{e}}$ ), interdigitation should be dominated by chain ends. This process has been analysed by various groups [5-7], with one hidden (and unrecognized) assumption : namely that the original distribution of terminal groups is uniform. This has been reexamined recently [8] : data on the molecular weight dependence of the surface tension of bulk polymers suggest that the surface density 


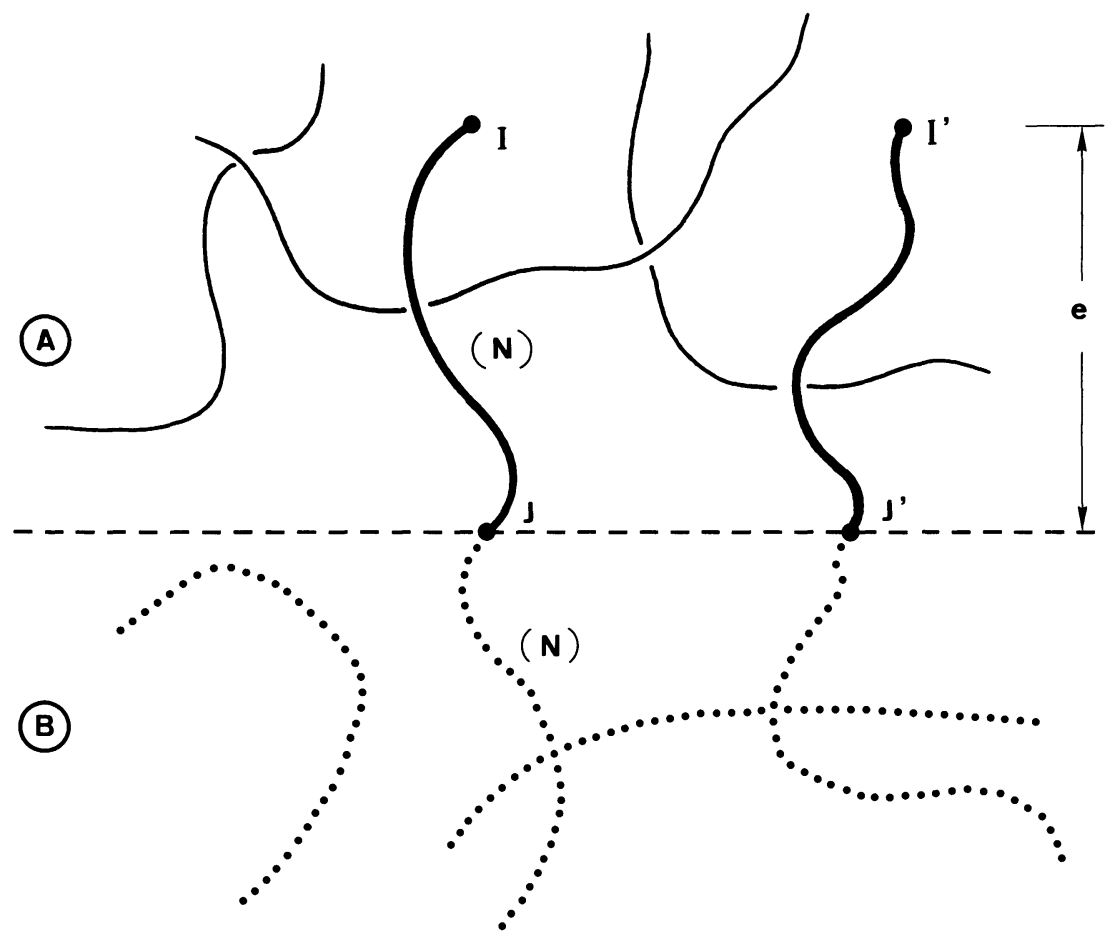

Fig. 3. - Adhesion between two glassy polymers (A) and (B) mediated by an AB block copolymer. The number $\nu$ of copolymers per unit contact area is assumed to be relatively low, so that each copolymer portion (IJ) is mainly surrounded by matrix chains. The length of the matrix chains (A or B) is also assumed to be much larger than the length $(N a)$ of each copolymer piece.

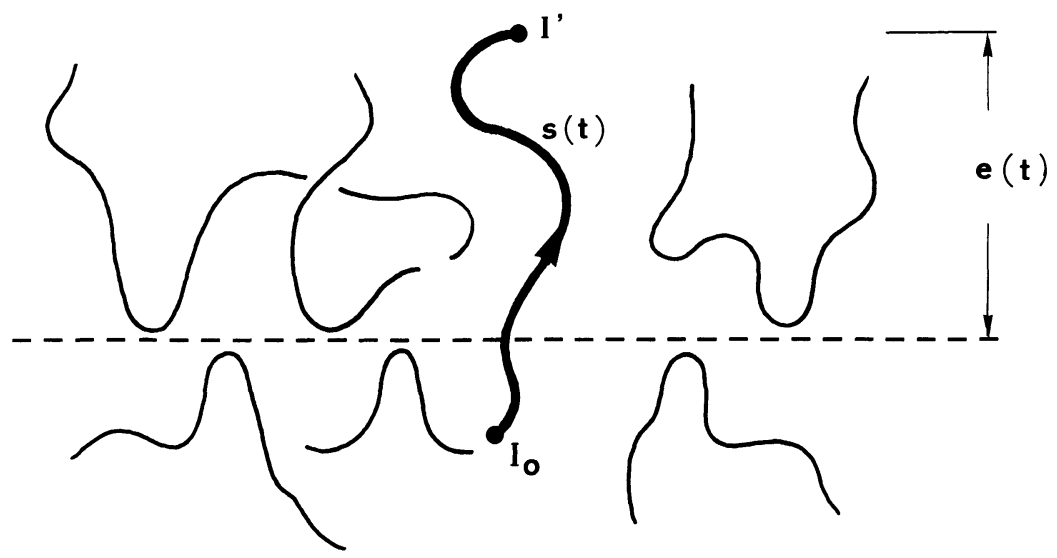

Fig. 4. - Partial interdigitation at an A/A contact. In an entangled regime, the chains reptate, during the healing time $t$, by a contour length $s(t)$, giving a skin thickness $e(t)$. Notice that most chains « fold back » at the contact plane, and do not participate. 
$\phi_{\text {I }}$ of chain ends is often anomalously high (of order $N^{-1 / 2}$ rather than $N^{-1}$, if we follow the arguments of reference [8]). Then for $t_{\mathrm{h}}<T_{\text {rep }}$, we should put

$$
\nu=\Phi_{\mathrm{I}} a^{-2} \approx N^{-1 / 2} a^{-2}
$$

where $a$ is a monomer size. This renormalisation of $\nu$ deeply changes the mechanical predictions. At first, for all these cases, one would expect that the fracture energy $G_{\text {IC }}$ is the product of $\nu$ (the number of bridges) by some characteristic work, either for chemical scission, or for mechanical succion of one bridging chain (Figs. 5, 6). This line of approach was used in references [7,9-11] : it is delicate, however, because it ignores many features associated with fracture mechanics. Our aim in the present text is to incorporate these features. In section 2 we construct the succion model, assuming that the succion takes place at constant density (no crazing). In section 3 we look at the mechanical aspects : they

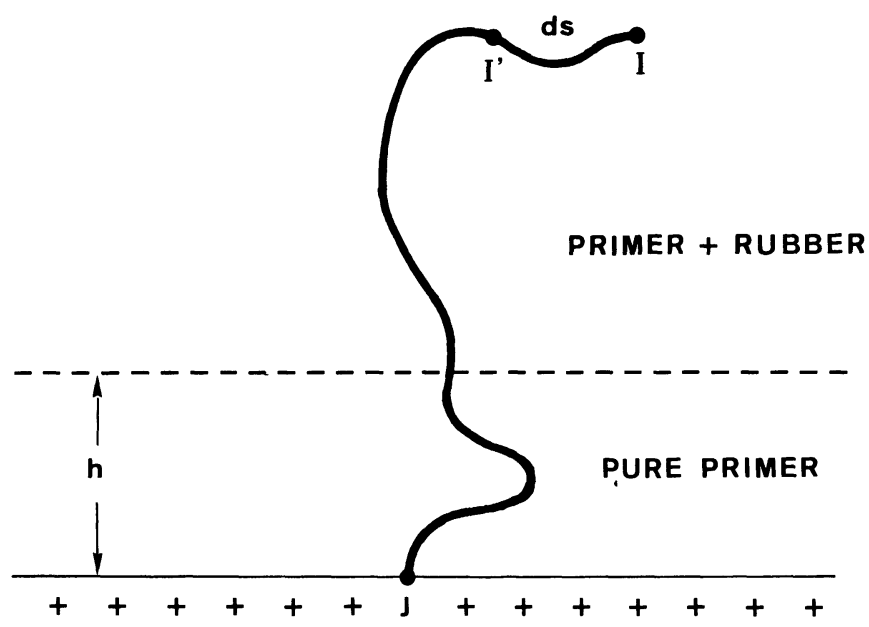

Fig. 5. - The succion process for the glass/rubber interface of figure 1 - assuming that the primer chains IJ break out of the rubber at their extremity $I$.
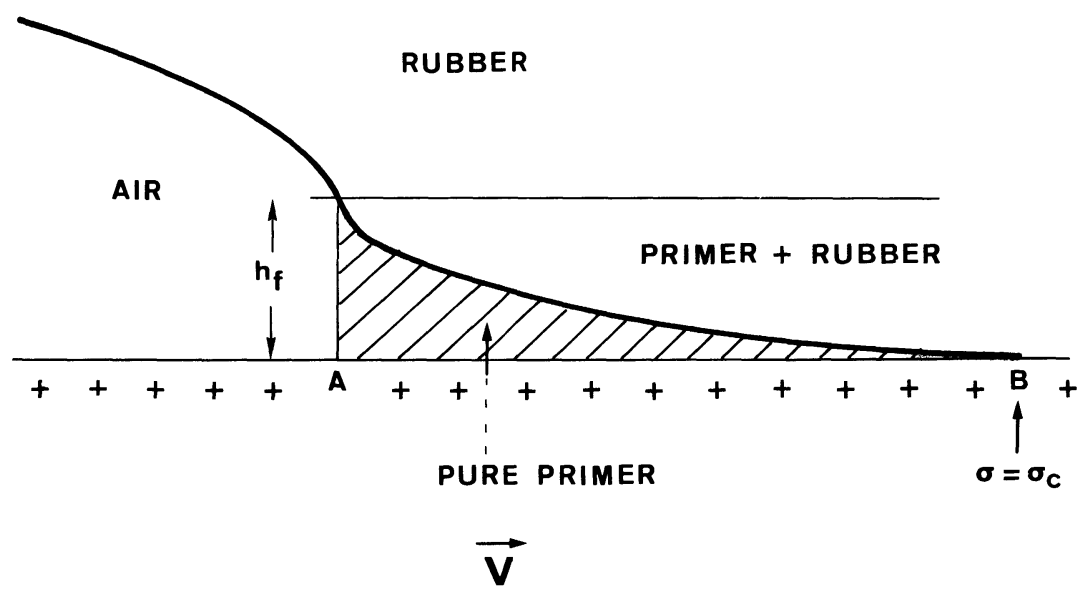

Fig. 6. - A more global view of the advancing fracture for the glass rubber interface of figure 1 . 
should in principle be analysed by Muskelishvili techniques. Here our aim is much more modest : we try to guess some simple scaling laws for the structure of the fracture region. Finally, in section 4, we return to a discussion of the different polymer systems. All this is extremeley tentative - but not far, in spirit, from many Friedel studies on dislocation motion (although the objects are very different !): thus it is bold, but not entirely unreasonable, to propose these conjectures here.

\section{The succion process.}

2.1 THE IDEAL CASE. - Consider, for instance, the glass/rubber interface of figure 1, assuming that chemical rupture takes place at the end I of the primer. Originally, the I points are distributed over a certain «skin thickness » $e$ : for a detailed theoretical discussion of $e$, see reference [12]. After rupture $\left(\sigma>\sigma_{\mathrm{c}}\right)$, the primer chains are progressively sucked out of the rubber (Fig. 5). If we look at the whole fracture region (Fig. 6), the process starts at point A (when $\sigma=\sigma_{\mathrm{c}}$ ). At a later stage (point $\mathrm{M}$ ), we find a layer of pure primer, with thickness $h$, covered by a mixed layer, of thickness $h-e$. Ultimately (at point $\mathrm{B}$ ), all the primer is extracted $\left(h=h_{\mathrm{f}}\right)$; the only remaining cohesion is due to the weak capillary forces : rupture takes place.

We assume no cavitation and no volume dilation between point $\mathrm{A}$ and point $\mathrm{B}$. When a chain is sucked in by a length $(\mathrm{d} s)$, the volume increase of the primer region is (ds $a^{2}$ ) where $a^{2}$ is the cross sectional area of a chain. Thus

$$
\mathrm{d} h=\nu a^{2} \mathrm{~d} s .
$$

Equivalently $h_{\mathrm{f}}=\nu a^{2} L$, where $L=N a$ is the extended length of the chains. It is helpful to visualize $h_{\mathrm{f}}$ as the volume extracted per unit area of fracture : the product of the volume per chain $N a^{3}$ by the number of chains $v$ per unit area.

After writing this balance of volumes, let us also write a balance of energy, in terms of the sucking force $f_{1}$ on one chain (at point $\mathbf{J}$ ). The work done per unit area is

$$
\sigma \mathrm{d} h=\nu f_{1} \mathrm{~d} s
$$

and inserting equation (2.1), this leads to

$$
a^{2} \sigma=f_{1}
$$

independent of $\nu$.

Let us now introduce a tube friction $\zeta_{\mathrm{t}}$ for one complete primer chain ( $N$ monomers) ; this will increase linearly with $N$ (see Ref. [4])

$$
\zeta_{\mathrm{t}}=\zeta_{1} N
$$

Here, however, only a fraction

$$
\Phi=\frac{h_{\mathrm{f}}-h}{h_{\mathrm{f}}}
$$

of the chain length is still to be sucked, and the relevant friction constant is $\Phi \zeta_{\mathrm{t}}$. This leads to a relation for the sucking force

$$
f_{1}=\zeta_{\mathrm{t}} \Phi \frac{\mathrm{d} s}{\delta \tau}
$$


Switching from the microscopic variables $\left(s, f_{1}\right)$ to the macroscopic variables $(h, \sigma)$ and using equations (2.1-3), we arrive at the succion law

$$
\sigma=Q \Phi \frac{\mathrm{d} h}{\mathrm{~d} t}=Q \frac{h-h_{\mathrm{f}}}{h_{\mathrm{f}}} \frac{\mathrm{d} h}{\mathrm{~d} t}
$$

with

$$
Q=\frac{\zeta_{\mathrm{t}}}{\nu a^{4}} .
$$

The friction constant $Q$ is the central element of our description of weak junctions.

2.2 EFFECTS OF CHAIN RETURN. - The rubber/glass interface is a simple example, because, in the initial state, the primer chains are always mixed with the rubber. The same remark holds for the [A polymer/AB copolymer/B polymer] system of figure $3:$ the A portion of a copolymer chain never enters the $B$ regions. However, if we look at the two other cases (Figs. 2 and 4), we see that a bridging chain may perform complicated zig zags between the two sides of the junction. Returning to figure 5 , we find that, if point $I$ is above the fracture plane, indeed the motion $\mathrm{I} \rightarrow \mathrm{I}^{\prime}$ gives a sucked volume $a^{2} \mathrm{~d} s$. But, if $\mathrm{I}$ is below the fracture plane, the motion $I \rightarrow I^{\prime}$ induces no volume change. This will occur roughly half of the time : thus we expect equations (2.1) and (2.2) to be modified by coefficients of order $1 / 2$ : but their scaling structure remains probably the same.

Thus we believe that (apart from numerical coefficients), the basic succion equation (2.7) may retain it's meaning for all four cases.

2.3 The SUCCION TIME. - Integrating equation (2.7) at fixed $\sigma$, we find a total time for succion $\left(h=h_{\mathrm{f}}\right)$ :

$$
\tau=\frac{h_{\mathrm{f}} Q}{2 \sigma} .
$$

This is the «duration of the experiment " which was introduced in section 1 , and which may be inserted into equation (1.2) defining the chemical stress $\sigma_{\mathrm{c}}$. Note that a similar self consistency equation holds for the plastic stress in glassy polymers - since, here also, the rates increase exponentially with $\sigma$.

\section{Fracture.}

3.1 General features (Fig. 7). - Far from the fracture tip (al all velocities $V$ much smaller than the sound velocity), we expect the standard scaling laws for a crack in an elastic medium of shear modulus $\mu$ :

$$
\begin{gathered}
\dot{\sigma} \cong K x^{-1 / 2} \\
u \cong K \mu^{-1} x^{1 / 2}
\end{gathered}
$$

for the stress $\sigma$ and the displacement $u$, at a distance $x$ from the source. The product $\sigma u$ is constant, and measures the energy $G$ per unit area of fracture

$$
G \cong \sigma u \cong K^{2} / \mu
$$

We have a weakened junction in the interval $\mathrm{AB}$ with a length

$$
x_{1}=\mathrm{AB}=V \int_{0}^{h_{\mathrm{f}}} \frac{\mathrm{d} t}{\mathrm{~d} h} \mathrm{~d} h
$$




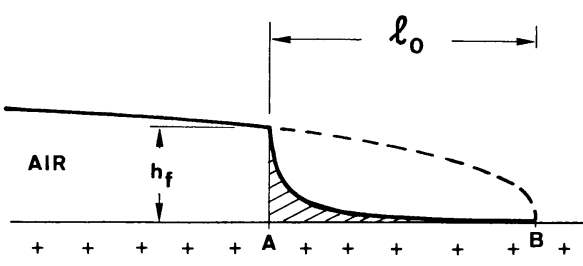

(a)

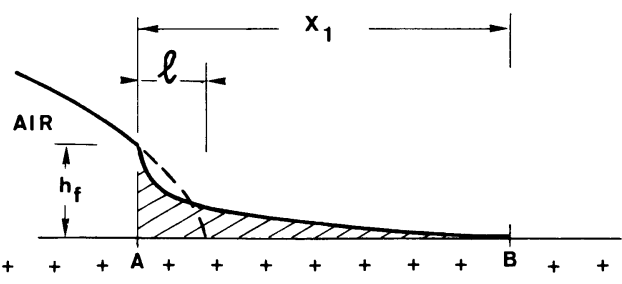

( $\beta$

Fig. 7. - Two regimes in the fracture process : $\alpha$ ) slow regime : the stresses at the tip adjust below threshold $\left(\sigma(\mathrm{A})=\sigma_{\mathrm{c}}-\varepsilon\right)$ and ensure that the junction length $\left(\tilde{x}_{1}\right)$ remains equal to the smearing length $\left.\left(\ell_{0}\right) ; \beta\right)$ fast regime : succion takes place in a large length $x_{1}$.

where $\mathrm{d} h / \mathrm{d} t$ is proportionnal to the local stress $\sigma$ (Eq. (2.7)). It turns out that the integral (3.4) is always dominated by the region of lower $\sigma$ (the slower portions). Here the lowest stress is at point $\mathrm{B}: \sigma(\mathrm{B})=\sigma_{\mathrm{c}}$. Thus

$$
x_{1} \cong V \tau\left(\sigma_{c}\right)
$$

where $\tau\left(\sigma_{\mathrm{c}}\right)$ is the succion time defined in equation (2.9). One last condition must be imposed : namely that the displacement $u(\mathrm{~A}) \cong h_{\mathrm{f}}$. This also leads to a scaling law for the fracture energy :

$$
G=\sigma(A) h_{\mathrm{f}} .
$$

The sharp singularity described in equations $(3.1,2)$ is in fact smeared out in the fracture region over a certain distance $\ell$. $\ell$ is related to $\sigma(A)$ by a condition of continuity for the stress near $\mathrm{A}$ :

$$
\sigma(A) \cong \mu \frac{\partial u}{\partial x} \cong \mu \frac{u(\mathrm{~A})}{\ell} \cong \mu \frac{h_{\mathrm{f}}}{\ell} .
$$

3.2 The SLOW REGiME. - Let us assume for a moment that $x_{1} \ll \ell$ : this means that, in the weakened portion $\mathrm{AB}$, the stress is nearly constant. Thus

$$
\sigma(\mathrm{A}) \cong \sigma(\mathrm{B})=\sigma_{\mathrm{c}}
$$

and

$$
\ell=\ell_{0} \equiv \frac{\mu}{\sigma_{\mathrm{c}}} h_{\mathrm{f}} .
$$

However, at this point, we face a major difficulty: the stress distribution is smeared out (when compared to an ideal fracture) over a length $\ell_{0}$. But the displacement field $u(x)$ is smeared out over a smaller length $x_{1}$. This is not acceptable : the two fields are related to one same smearing function $\phi(x)$, extending from 0 to $x_{1}$. The explicit forms are (ignoring all numerical coefficients)

$$
\begin{aligned}
& \sigma(x)=\mu \int_{0}^{x} \phi(y) \frac{1}{(x-y)^{1 / 2}} \mathrm{~d} y \\
& u(x)=\int_{x}^{x_{1}} \phi(y)(x-y)^{1 / 2} \mathrm{~d} y .
\end{aligned}
$$


And thus the smearing length must be equal to $x_{1}$ (within numerical coefficients).

The physical answer to this paradox seems to be the following: the succion time $\tau(\sigma)$ is a very steep function of the applied stress. Up to now we took

$$
\tau= \begin{cases}\tau\left(\sigma_{\mathrm{c}}\right) & \sigma \geqslant \sigma_{\mathrm{c}} \\ \infty & \sigma<\sigma_{\mathrm{c}} .\end{cases}
$$

However, if $\sigma$ is slightly below $\sigma_{\mathrm{c}}, \tau$ is actually long, but not infinite. (For instance, in a glassy polymer, plastic yield corresponds to a $\tau$ which depends exponentially on $\sigma$ ). Thus, by a very small shift of the stress

$$
\sigma(\mathrm{A})=\sigma_{\mathrm{c}}-\varepsilon
$$

we can arrange that $\tau$ is suitably increased, so that the length $\tilde{x}=V \tau$ becomes equal to $\ell_{0}$. Then our system of stresses and strains is compatible. The final conclusion is simple : returning to (3.6) we see that the fracture energy reduces to

$$
G_{0}=\left(\sigma_{\mathrm{c}}-\varepsilon\right) h_{\mathrm{f}} \approx \sigma_{\mathrm{c}} h_{\mathrm{f}} .
$$

In 1982, I had made a different conjecture [9] : $G_{0}=\sigma_{\mathrm{c}} L_{\mathrm{b}}$ (where $L_{\mathrm{b}}$ is the contour length of the sucked chains. But equation (3.10) is preferable, at least for situations without cavitation : it is a consequence of our improved analysis of volume and energy balance in the succion process (Eqs. (2.1-3) above).

The slow regime holds up to a certain characteristic velocity $V^{*}$ such that the unperturbed $x_{1}$ becomes comparable to $\ell_{0}$ : comparing (3.5) and (3.9), we see that

$$
V^{*}=\frac{\ell_{0}}{\tau} \cong \frac{\mu}{Q} .
$$

3.3 CONJECTURES ON THE FAST REGIME $\left(V>V^{*}\right)$. - Here we postulate a two scale form for the smearing function $\phi(x)$ [defined after Eq. (3.9)] :

a) on a relatively small interval $0<x<\ell$ (where $\ell$ may be different from $\ell_{0}$ ), $\phi(x)$ is high ;

b) on a larger interval $\ell<x<x_{1} \phi(x)$ is small but non vanishing (to ensure a finite opening $u(x)$ of the junction).

We do not know whether it is indeed possible to satisfy the junction condition (2.7) at all points $0<x<x_{1}$ with this type of function $\phi(x)$. But if we do make the assumptions (a) and (b), we are led to the following conclusions: in the interval $(0, \ell)$ the stress is high $\sigma \approx \sigma(\mathrm{A})>\sigma_{\mathrm{c}}$. Since most of the fracture singularity is in this region, we may relate $\sigma(\mathrm{A})$ to the stress at the other end of the junction $\sigma(\mathrm{B})=\sigma_{\mathrm{c}}$ using equation (3.1) :

$$
\frac{\sigma^{2}(\mathrm{~A})}{\sigma_{\mathrm{c}}^{2}}=\frac{x_{1}}{\ell} .
$$

Note that $\ell$ is now velocity dependent $\left(\ell \neq \ell_{0}\right)$. However, we can eliminate $\ell$ between (3.12) and (3.7). We then arrive at

$$
\sigma(\mathrm{A})=\sigma_{\mathrm{c}} \frac{V}{V^{*}}
$$

Equation (3.6) then gives the fracture energy

$$
G(V) \cong G_{0} \frac{V}{V^{*}}
$$


The characteristic length $\ell$ (derived from Eqs. $(3.7,13)$ ) is

$$
\ell=\frac{\mu h_{\mathrm{f}}}{\sigma(\mathrm{A})}=\frac{\mu h_{\mathrm{f}}}{\sigma_{\mathrm{c}}} \frac{V^{*}}{V} .
$$

Of course these conjectures should be supported by a detailed calculation of the smearing function $\phi(x)$ (defined after Eq. (3.9)).

\section{Discussion.}

4.1 SUMMARY OF RESUlTS. - In situations without cavitation, our weak junctions are described by three main parameters :

(a) the threshold stress $\sigma_{\mathrm{c}}$ - controled either by chemical breakdown (in rubbers) or by plastic flow (in glassy polymers). $\sigma_{c}$ is independent of the density of bridges $(\nu)$ and also not dependent on the length of the bridges $L_{\mathrm{b}}$;

(b) the volume (per unit area) of sucked material $h_{\mathrm{f}}=\nu a^{2} L_{\mathrm{b}}$. For fixed $\nu$ this would be a linear function of the bridge molecular weight. But in most practical cases, $\nu$ will be smaller at large $L_{b}$ : for instance on the glass/rubber interface, it is difficult to graft long primers in a dense way ;

(c) the friction constant $Q$ describing the succion process (Eq. (2.7)) or equivalently the succion time $\tau\left(\sigma_{\mathrm{c}}\right)$ (Eq. (2.9)). Equation (2.8) shows that $Q$ is proportionnal to $L_{\mathrm{b}} / \nu$.

4.2 RUBBER/RUBBER JUNCTIONS (Fig. 1). - For a rubber/rubber junction - with an average number $N$ of monomers between cross links in the bulk rubber - we expect $L_{\mathrm{b}} \approx N a$ and $\nu \approx \nu_{\mathrm{p}} \approx N^{-1 / 2} a^{-2}$. Here $h_{\mathrm{f}} \approx N^{1 / 2} a$ and $Q \approx N^{3 / 2}$. The modulus $\mu$ for this case is of order $k T /\left(N a^{3}\right):$

a) the quasistatic fracture energy is predicted to be

$$
G_{0}=\sigma_{\mathrm{c}} h_{\mathrm{f}}+\nu_{\mathrm{c}} u+2 \gamma
$$

where we have added (for completeness) the corrections due to chemical rupture ( $U$ is the energy for disruption of one bond) and to the surface tension $\gamma$.

We expect the leading term to be $\sigma_{\mathrm{c}} h_{\mathrm{f}}$ : this term scales like $N^{1 / 2}$. It shows a qualitative trend observed long ago by Gent and coworkers [14] : namely that upon increasing the density of cross links (decreasing $N$ ) the energy $G_{0}$ decreases. This explanation differs profoundly from the old argument of Lake and Thomas [15].

However, there are many complications, which must be emphasized : if we go to very weak cross linking in the bulk rubbers, we produce long dangling ends, giving strong viscoelastic effects far out from the junction region, as first shown by Gent and Petrich [16]. This regime is qualitatively discussed in reference [2], but is outside the scope of the present discussion;

b) on dynamical features : equation (2.9) shows that the succion time $\tau$ scales here like $N^{2}$. The characteristic velocity $V^{*}$ derived from equation (3.11) is

$$
V^{*}=\frac{k T}{a \zeta_{1}} N^{-5 / 2}
$$

where $\zeta_{1}$ is a monomer friction coefficient. In a rubber (well above $T_{\mathrm{g}}$ ), we expect

$$
\frac{k T}{a \zeta_{1}} \approx 1 \text { to } 10 \text { meters } / \mathrm{s} \text {. }
$$


Taking $N=30$, this would correspond to $V^{*}$ values between $0.2 \mathrm{~mm} / \mathrm{s}$ and $2 \mathrm{~mm} / \mathrm{s}$.

It is amusing to note that Kramer and Berger, measuring the growth velocity of crazes (at fixed final elongation) in polystyrene, found an empirical law $V(N)$ - where $N$ is now the D.P. of the linear chains - which looks exactly like equation (2.2). However, the two problems are different. Remember in particular that our discussion assumes no cavitation and thus no crazing. Some effects of cavitation are discussed in a separate note [18].

4.3 The CASE OF PARTIAL INTERDifFusion (Fig. 4). - Here, the parameters $\nu$, $h_{\mathrm{f}}, Q$ depend on the healing time $t$. We shall discuss qualitatively in the limit of short healing ( $t$ smaller than the reptation time of the bulk material). The number of monomers per chain will be called $N$ and we assume that the chains are strongly entangled (we consider only cases where the entanglement distance $N_{\mathrm{c}}$ is small : of order of a few units).

Interdiffusion then takes place by reptation [5-7] (Fig. 8). The contour length of the portions which have penetrated is of order

$$
L_{\mathrm{b}}(t) \cong s(t)=\left(D_{\text {tube }} t\right)^{1 / 2}
$$

where $D_{\text {tube }}=k T / \zeta_{1}$ is the tube diffusion coefficient and $\zeta_{\mathrm{t}}=\zeta_{1} N$ is the tube friction constant. The skin thickness (i.e. the width of our weak junction) is [5] :

$$
e(t) \cong[\text { as }(t)]^{1 / 2}\left(\approx t^{1 / 4}\right) \text {. }
$$

The main problem is to determine the density of bridges $\nu$. Here we have to distinguish two cases :

4.3.1 Ideal distribution. - If the number of chain ends per unit volume $C_{\mathrm{I}}$ was uniform $\left(C_{\mathrm{I}}=2 / N a^{3}\right)$, we may put

$$
\nu=C_{\mathrm{I}} e(t) \cong \frac{e(t)}{N a^{3}}\left(\approx t^{1 / 4}\right) .
$$

4.3.2 Extremities attracted towards the free surface. - Here the original distribution of extremities had a $\delta$ function peak at the surface $(z=0)$

$$
C_{\mathrm{I}}=\nu_{\mathrm{I}} \delta(z)
$$

and the surrounding regions are strongly depleted in the original state. During healing, the peak described in equation (4.5) broadens and has a width $e(t)$, but it's integral strength $\int C_{\mathrm{I}} \mathrm{d} z$ stays unchanged. Then $\nu=\nu_{\mathrm{I}}$ is independent. In the most extreme case, discussed in reference [8], all the chain extremities within a distance $R_{0}=N^{1 / 2} a$ from the junction plane are originally captured on this plane : this gives

$$
\nu_{1} \cong N^{-1 / 2} a^{-2} \text {. }
$$

We can now discuss the fracture energy $G$, which was measured by the Lausanne group. The relevant value of the velocity $V$ is not quite clear in these experiments; we shall consider here only the static limit $V<V^{*}$ where equation (3.10) holds. This gives

$$
G(t) \cong \sigma_{\mathrm{c}} h_{\mathrm{f}} \cong \sigma_{\mathrm{f}} \cong \sigma_{\mathrm{c}} \nu a^{2} s(t) .
$$

In the ideal case, and contrary to common belief $[5,7]$, this would lead to

$$
G(t) \approx \sigma_{\mathrm{c}} \frac{e s}{a} \approx t^{3 / 4} .
$$


On the other hand, when the chain ends are originally trapped on the surface, we get

$$
G \approx \sigma_{\mathrm{c}} \nu_{\mathrm{I}} a^{2} s(t) \approx t^{1 / 2}
$$

in better agreement with the experiments [3]. However, the velocity conditions should be understood more deeply before any final conclusion can be reached.

4.4 WORDS OF CAUTION. - Our discussion of weak junctions is relatively simple, but fragile from various standpoints.

a) the mechanical analysis of section 3 may be completely wrong;

b) the possible role of cavitation and crazing in glassy polymers is ignored ;

c) how weak must the junction be for our model to apply ? We should study the viscoelastic losses in the surrounding medium (i.e. make the modulus $\mu$ complex $\mu \Rightarrow \mu^{\prime}+\mu^{\prime \prime}$ ) and compare them to the losses in the junction, described by the friction constant $Q$. At low bridging $(\nu \Rightarrow 0)$, the junction losses should dominate, but this idea should be transformed into a quantitative criterion.

It is tempting (and indeed it has been done) to discuss the «green strength » of a bulk, uncrosslinked, glassy polymer in the spirit of section $4.2-$ i.e. to keep the same picture for long healing times, where we do not really have a weak junction. This situation will clearly require a separate study. Some aspects, incorporating a possible role of cavitation, are discussed in reference [18].

\section{Acknowledgements.}

I have greatly benefited from correspondence and discussions on related subjects with A. N. Gent, D. Tabor, D. Maugis and L. Monnerie. Preprints from A. Donald, E. Kramer and R. Wool have also been extremely helpful.

\section{References}

[1] Kinloch A. J., Young R. J., Fracture behavior of polymers (Elsevier) 1983.

[2] De Gennes P. G., C.R. Acad. Sci. Paris II 307 (1988) 1949-1953.

[3] Jud K., Kausch H., Williams J. G., J. Mater. Sci. 16 (1981) 204-230 ;

Kausch H., Pure Appl. Chem. 55 (1983) 833-860.

[4] See for instance DE GENNES P. G., Scaling concepts in polymer physics (Cornell UP) 1985.

[5] De Gennes P. G., C.R. Acad. Sci. Paris B 291 (1980) 219-222.

[6] Prager S., Tirrell M., J. Chem. Phys. 75 (1981) 5194.

[7] Wool R., Connor K. O., J. Appl. Phys. 52 (1981) 5953-5960 ; J. Polymer Sci. Lett. 20 (1982) 710 ;

KIM Y. H., Wool R., Macromolecules 16 (1983) 1115-1122.

[8] De Gennes P. G., C.R. Acad. Sci. Paris II 307 (1988) 1841-1844.

[9] DE GenNes P. G., in Microscopic aspects of adhesion and lubrication, Ed. J. M. Georges (Elsevier) 1982.

[10] Prentice P., Polymer 24 (1983) 344-350.

[11] Mcleish T., Plummer C., Donald A. M. (to be published).

[12] DE Gennes P. G., Macromolecules 13 (1980) 1069-1075.

[13] Kanninen M. K., Popelar C. H., Advanced Fracture Mechanics (Oxford) 1985.

[14] Gent A. N., Adhesives'age 25 (1982) 27-35.

[15] Lake G., Thomas A., Proc. R. Soc. London A 300 (1967) 108-119.

[16] Gent A. N., Petrich R., Proc. R. Soc. A 310 (1969) 433-450.

[17] Berger L., Kramer E., Macromolecules 20 (1987) 1980-1985.

[18] De Gennes P. G., C.R. Acad. Sci. Paris (à paraître). 